\title{
mTORC1 and mTORC2 Expression Levels in Oral Squamous Cell Carcinoma: An Immunohistochemical and Clinicopathological Study
}

\author{
GORO KAWASAKI, TOMOFUMI NARUSE, KOHEI FURUKAWA and MASAHIRO UMEDA \\ Department of Clinical Oral Oncology, Unit of Translational Medicine, \\ Nagasaki University Graduate School of Biomedical Sciences, Nagasaki, Japan
}

\begin{abstract}
Background/Aim: Mammalian target of rapamycin ( $m$ TOR) plays a critical role in the regulation of tumor cell motility, invasion and cancer cell metastasis. mTOR consists of two separate multi-protein complexes, mTOR complex (mTORC) 1 and mTORC2. Materials and Methods: We investigated the expression levels of mTORC1 and mTORC2 immunohistochemically in oral squamous cell carcinoma (OSCC). Results: mTORC1 and mTORC2 were more highly expressed in tumors than in normal oral mucosa. mTORC1 expression was correlated with $T$ classification, $N$ classification, and survival rate $(p<0.05)$, whereas mTORC2 expression was only correlated with $T$ classification $(p<0.05)$. Histologically, the expression levels of mTORC1 and mTORC2 correlated with cancer cell invasion and the expression of proliferating cell nuclear antigen $(p<0.05)$, respectively. Expression levels of vascular endothelial growth factors and hypoxia-inducible factor 1 in the mTORC1 (-)/ mTORC2 (+) group were significantly lower than those in other groups. Conclusion: These findings suggested that mTORCl and mTORC2 could be promising anti-tumor targets in OSCC, and mTORC1 (-)/mTORC2 (+) may have a correlation with the malignant potential of OSCC.
\end{abstract}

Cancer cell migration and invasion play fundamental roles in cancer metastasis. Mammalian target of rapamycin (mTOR) is a $289-\mathrm{kDa}$ serine/threonine kinase, belonging to the phosphoinositide 3-kinase (PI3K)-related kinase family, that regulates cell growth, proliferation, and progression of the cell cycle (1). Recent studies have shown that mTOR

Correspondence to: Goro Kawasaki, DDS, PhD, Department of Clinical Oral Oncology, Unit of Translational Medicine, Nagasaki University Graduate School of Biomedical Sciences, 1-7-1, Sakamoto, Nagasaki, 852-8588, Japan. Tel: +81 958497696, Fax: +81958497700, e-mail: gkawa@nagasaki-u.ac.jp

Key Words: mTORC1, mTORC2, oral cancer. also plays a critical role in the regulation of tumor cell motility and invasion and cancer cell metastasis $(2,3)$.

mTOR is activated by the phosphorylation of Ser2448 through the PI3K/Akt signaling pathway, which then completes its functions by activating p70 ribosomal S6 kinase and phosphorylating the eukaryotic initiation factor $4 \mathrm{E}$ binding protein $1(4,5)$. The mTOR pathway and mTOR genes were discovered while investigating the mechanism of action of their inhibitor rapamycin and the causes of resistance (6). Rapamycin, a macrolide antibiotic product, was first discovered in 1975 from samples collected on Easter Island in the South Pacific (6). A few years later, rapamycin demonstrated significant immunosuppressive activity in preventing the development of allergic encephalomyelitis and adjuvant arthritis in rats. The basis of its immunosuppressive properties was established by many later studies and led to the establishment of rapamycin as a major immunosuppressant against transplant rejection (6).

mTOR consists of two separate multi-protein complexes, mTOR complex (mTORC) 1 and mTORC2, which are both activated by growth factor stimulation $(6,7)$. mTORC1 phosphorylates p70 S6 kinase (S6K1) and eukaryotic initiation factor $4 \mathrm{E}$ (eIF4E) binding protein 1 (4E-BP1), and regulates cell growth, proliferation, survival, and motility (8). mTORC2, which phosphorylates Akt, protein kinase $\mathrm{C}$, and focal adhesion proteins, controls the activities of small GTPases, and regulates cell survival and the actin cytoskeleton (8). Rapalogs are first-generation mTOR inhibitors and they allosterically inhibit mTORC1 but not mTORC2 $(9,10)$. Activated mTOR has been associated with poor prognosis in various cancers, including oral squamous cell carcinoma (OSCC) (11-14). However, the anti-tumor effects of mTOR inhibitors in OSCC remain unclear. To ascertain the effects of mTOR inhibitors in OSCC, it is important to investigate the expression levels of mTORC1 and mTORC2 in OSCC.

In the present study, we investigated immunohistochemically the expression levels of mTORC1 and mTORC 2 in OSCC and examined their relationships with clinical and pathological factors. 


\section{Materials and Methods}

Paraffin-embedded sections were obtained from biopsy specimens from 72 patients with OSCC who underwent radical surgery in our department between January 2000 and December 2007. The tumor stage was classified according to the TNM classification of the International Union Against Cancer (15). The histological differentiation of tumors was defined according to the WHO classification (16) and the invasive grade was assessed by the Yamamoto-Kohama (YK) mode of invasion (17).

Deparaffinized sections in xylene were soaked in $10 \mathrm{mmol} / \mathrm{l}$ citrate buffer ( $\mathrm{pH} 6.0$ ) and placed in an autoclave at $121^{\circ} \mathrm{C}$ for 5 min for antigen retrieval. Endogenous peroxidase was blocked by incubation with $0.3 \% \quad \mathrm{H}_{2} \mathrm{O}_{2}$ in methanol for $30 \mathrm{~min}$. Immunohistochemical staining was performed using an Envision system (ENVISION+; DAKO, Glostrup, Denmark). The primary antibodies used were against mTORC1, mTORC2, proliferating cell nuclear antigen (PCNA), vascular endothelial growth factor (VEGF)-A, VEGF-C, and hypoxia-inducible factor $1 \alpha$ (HIF-1 $\alpha$ ) (DAKO, Glostrup, Denmark). The sections were then washed in Dulbecco's phosphate buffered saline (PBS), followed by incubation with the primary antibodies at $4^{\circ} \mathrm{C}$ over night. The reaction products were visualized by immersing the sections in diaminobenzidine (DAB) solution, and the samples were counterstained with Mayer's hematoxylin and mounted.

Results were evaluated by calculating the total immunostaining score as the product of the proportional score and intensity score. The proportional scores described the estimated percentage of positively stained tumor cells $(0$, none; $1,<10 \% ; 2,10-50 \% ; 3,50-$ $80 \% ; 4,>80 \%)$. The intensity score represented the estimated staining intensity ( 0 , no staining; 1 , weak; 2 , moderate; 3 , strong). Total scores ranged from 0 to 12 . Immunohistochemical overexpression was defined as a total score of 4 or greater, because immunohistochemical expression levels in samples showed a bimodal distribution with the discriminating nadir at a total score value of 3 to 4 . mTOR (-) was defined as a total mTOR immunostaining score ranging from 0 to 3 and mTOR (+) was defined as total mTOR immunostaining score of 4 or greater.

The relationships between the sample expression of target molecules and clinicopathological features were assessed using Fischer's exact test. Survival analysis was calculated using the Kaplan-Meier method and compared using the log-rank test. A multiple regression study was performed using Cox's proportional hazard analysis and chi-squared test. $p$-Values $<0.05$ were considered to be significant.

\section{Results}

mTORC1 and mTORC2 were expressed mainly in the cytoplasm of the tumor cells (Figure 1). In OSCC specimens, mTORC1 and mTORC2 were detected in 37 tumors and 50 tumors, respectively. mTORC1 and $\mathrm{mTORC} 2$ protein expression was absent or minimal in the cytoplasm of epithelial cells in normal oral tissue. mTORC1 expression correlated with $\mathrm{T}$ classification, $\mathrm{N}$ classification, and survival rate $(p<0.05)$, whereas mTORC2 expression was only correlated with $\mathrm{T}$ classification $(p<0.05)$ (Table I). Histologically, the expression levels of mTORC1 and
mTORC2 correlated with cancer cell invasion by Y-K classification (Table II) and the expression of PCNA $(p<0.05)$ (Table III), respectively. Concerning the survival rate, there was significant difference between mTORC1(+) and mTORC1(-), but there was no significant difference between mTORC2(+) and mTORC2(-) (Figure 2).

The numbers of case with mTORC1 $(-) /$ mTORC2 $(-)$, mTORC1 (-)/mTORC2 (+), mTORC1 (+)/mTORC2 (-), and mTORC1 $(+) / \operatorname{mTORC} 2(+)$ were $16,17,6$, and 33 , respectively. In OSCC samples, VEGF-A, VEGF-C and HIF$1 \alpha$ protein expressions were detected 50,51 and 50 samples, respectively. VEGF-A, VEGF-C and HIF-1 $\alpha$ were expressed in cytoplasm of OSCC (Figure 1). The expression levels of VEGFs and HIF-1 $\alpha$ in the mTORC1 (-) mTORC2 (+) group were significantly lower than those in the other groups (Table IV).

\section{Discussion}

The anticancerous activity of rapamycin was first demonstrated in human glioma tumor xenografts in mice (6, 18). In the last few years, significant advances have been made in understanding the role of mTOR in cancer development and progression (3, 19). Increased mTOR signaling in cancers often occurs as a result of mutations in the pathways closely related to mTOR. Recently, activating mutations of mTOR itself have been identified through mining of human cancer genome databases (20). Activation of the $\mathrm{PI} 3 \mathrm{~K} / \mathrm{Akt} / \mathrm{mTOR}$ pathway through such mechanisms has been shown to correlate with tumor progression and reduced survival in patients across a variety of tumor types $(21,22)$.

To investigate the role in initiation and development of cancer, molecular approaches have been used to study the specific components of the mTOR pathway (3). Some researchers have demonstrated that molecular inhibition of mTOR, Rictor, or Raptor leads to a significant decrease in proliferation of cancer cells and attenuates cell cycle progression $(21,23,24)$.

mTOR also plays a key role in advanced and metastatic disease. Although the molecular mechanisms of the regulation of cell motility and metastasis by mTOR are not fully understood, there are some reports concerning metastasis in breast cancer, gliomas and head and neck cancer (25-27). In colorectal cancer, Gulhati et al. demonstrated that mTORC1 and mTORC2 are intimately involved in epithelial-mesenchymal transition, motility, and metastasis (26). In head and neck squamous cell carcinoma, Patel et al. (28) developed an orthotopic model of head and neck squamous cell carcinoma (HNSCC) consisting of the implantation of HNSCC cells into the tongue of immunocompromised mice, and they found that inhibition of mTOR with rapamycin and the rapalog RAD001 diminished lymphangiogenesis in the primary tumors and prevented the 

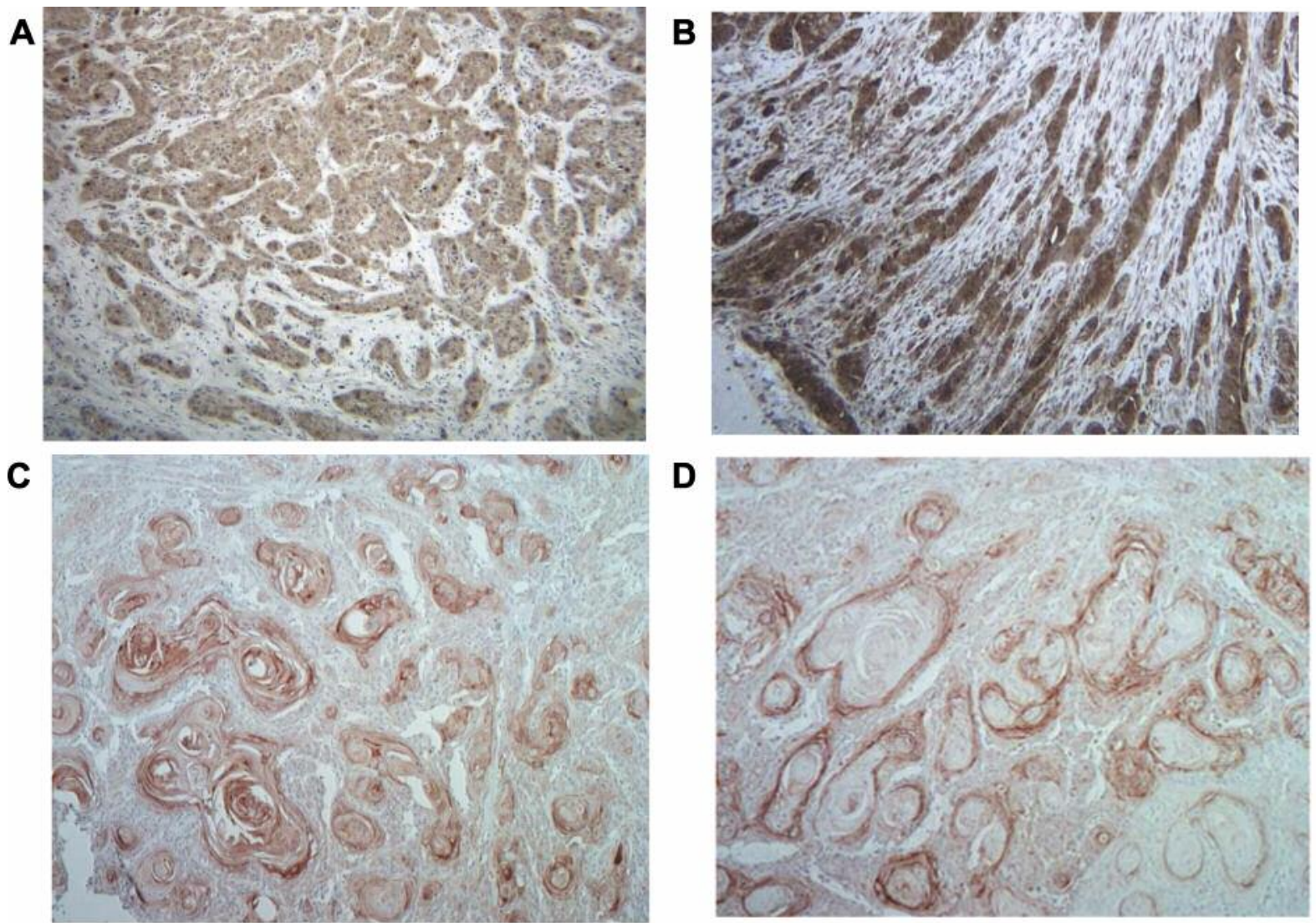

Figure 1. Immunohistochemically determined expression levels of mTORC1, mTORC2, VEGF-A and VEGF-C. Immunohistochemically-positive cells can be seen in the cancer cell nest. A; mTORC1, B; mTORC2. C; VEGF-A, D;VEGF-C.
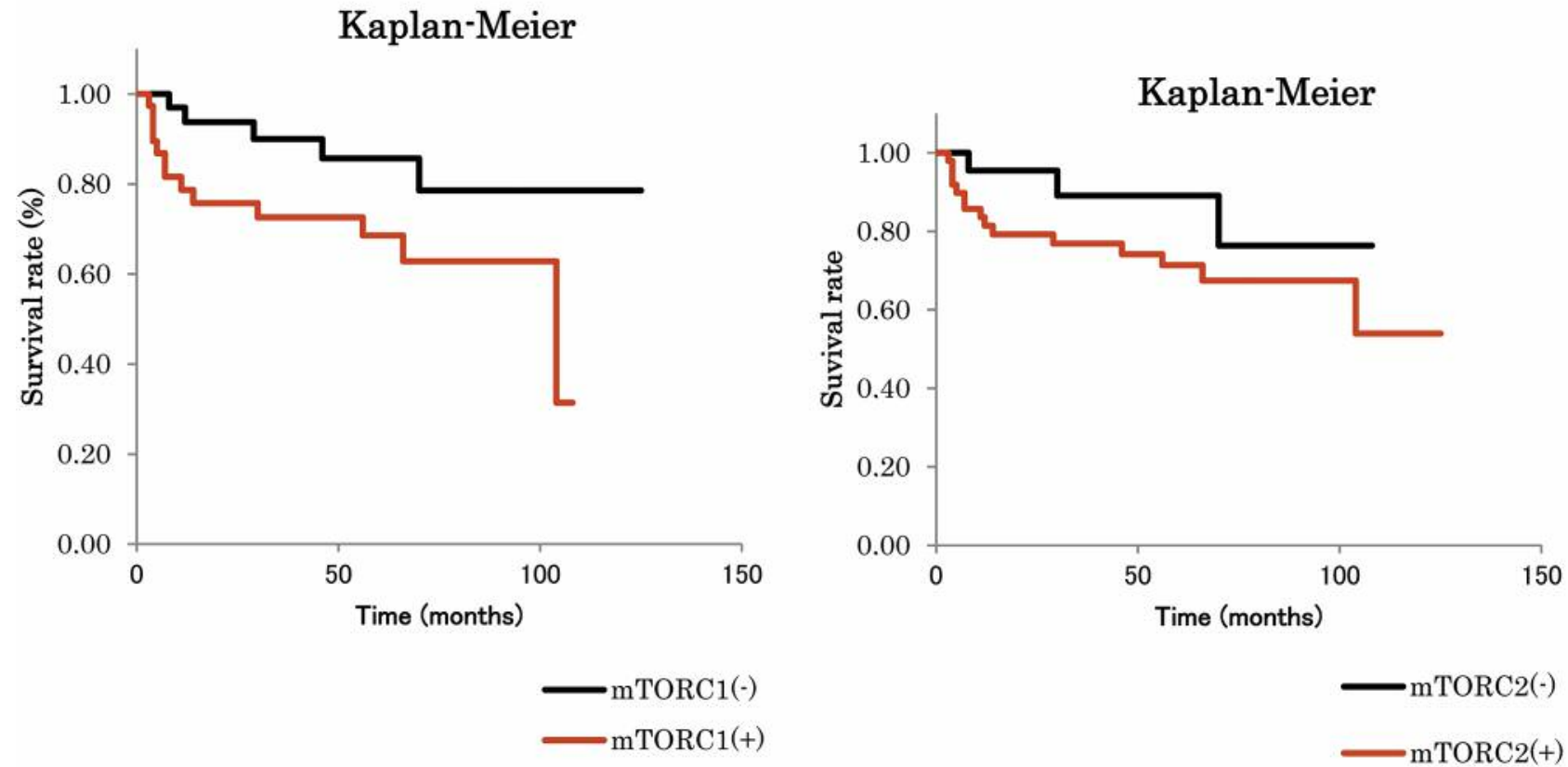

Figure 2. Kaplan-Meier survival curve. Significant differences in survival are present between the mTORC1 positive and negative groups. There is no significant difference between the mTORC2-positive and -negative groups. 
Table I. Correlation between mTORC1 and mTORC2 expression and clinicopathological features.

\begin{tabular}{|c|c|c|c|c|c|c|}
\hline & $\begin{array}{c}\operatorname{mTORC} 1(-) \\
(\mathrm{n}=33)\end{array}$ & $\begin{array}{c}\operatorname{mTORC} 1(+) \\
(\mathrm{n}=39)\end{array}$ & $p$-Value & $\begin{array}{c}\operatorname{mTORC2}(-) \\
(\mathrm{n}=22)\end{array}$ & $\underset{(\mathrm{n}=50)}{\operatorname{mTORC} 2(+)}$ & $p$-Value \\
\hline Gender & & & 0.475 & & & 0.61 \\
\hline Male & 21 & 21 & & 14 & 28 & \\
\hline Female & 12 & 18 & & 8 & 22 & \\
\hline Age & & & 0.806 & & & 0.79 \\
\hline $67 \geq$ & 11 & 15 & & 7 & 19 & \\
\hline $67<$ & 22 & 24 & & 15 & 31 & \\
\hline \multicolumn{7}{|l|}{$\mathrm{T}$ categories } \\
\hline $\mathrm{T} 1+\mathrm{T} 2$ & 29 & 24 & $p<0.05$ & 20 & 32 & $p<0.05$ \\
\hline $\mathrm{T} 3+\mathrm{T} 4$ & 4 & 15 & & 2 & 18 & \\
\hline \multicolumn{7}{|l|}{$\mathrm{N}$ categories } \\
\hline No & 30 & 24 & $p<0.05$ & 20 & 37 & 0.126 \\
\hline $\mathrm{N} 1+\mathrm{N} 2$ & 3 & 15 & & 2 & 13 & \\
\hline \multicolumn{7}{|l|}{ Histological grade } \\
\hline well & 31 & 35 & 0.42 & 21 & 45 & 0.659 \\
\hline moderate. poor. & 2 & 4 & & 1 & 5 & \\
\hline
\end{tabular}

Table II. Correlation between mTORC1 and mTORC2 expressions and YK mode of invasion of oral SCC.

\begin{tabular}{lccccc}
\hline Y-K classification & $\begin{array}{c}\text { mTORC1(-) } \\
\mathrm{n}=33\end{array}$ & $\begin{array}{c}\text { mTORC1(+) } \\
\mathrm{n}=39\end{array}$ & $p$-Value & $\begin{array}{c}\text { mTORC 2(-) } \\
\mathrm{n}=22\end{array}$ & $\begin{array}{c}\text { mTORC2(+) } \\
\mathrm{n}=50\end{array}$ \\
\hline Grade 1,2,3 & 27 & 23 & 20 & 30 \\
Grade 4C,4D & 6 & 16 & $<0.05$ & 2 & 20 \\
\hline
\end{tabular}

Table III. Correlation between mTORC1 and mTORC2 expressions and PCNA-LI.

\begin{tabular}{lcccccc}
\hline & mTORC1(-) & mTORC1(+) & $p$-Value & mTORC2(-) & mTORC2(+) & $p$-Value \\
\hline $\mathrm{n}$ & 33 & 39 & & 22 & 50 & \\
PCNA-LI & $23.4 \pm 20.7$ & $50.7 \pm 22.9$ & $p<0.05$ & $26.7 \pm 24.8$ & $43.3 \pm 24.8$ & $p<0.05$ \\
\hline
\end{tabular}

PCNA-LI: PCNA labelling index.

dissemination of head and neck cancer cells to the cervical lymph nodes. In addition, knockdown of mTORC1 and mTORC2 was reported to sensitize colorectal cancer cell lines to apoptosis induced by oxaliplatin (26).

mTORC1 is comprised of mTOR, regulatory-associated protein of mTOR (Raptor), mLST8/G $\beta$ L, Deptor, and prolinerich AKt substrate 40 (29). mTORC1 influences cell growth and proliferation by promoting the biosynthesis of proteins, lipids, and organelles, and by limiting catabolic processes (8). In addition, activation of mTORC1 is sufficient for tumorigenesis, such as the promotion of glycolysis, increased flux through the oxidative branch of the pentose pathway, and enhanced de novo lipogenesis (30). mTORC2 consists of mTOR, rapamycininsensitive companion of mTOR (Rictor), mLST8/G $\beta \mathrm{L}$, Protor, Deptor, and mammalian stress-activated protein kinase interacting protein (mSIN1) (8). Compared with mTORC1, the
Table IV. Correlation between VEGF and HIF- $\alpha$ expression and mTORC1/mTORC2 expressions.

\begin{tabular}{lccc}
\hline mTORC1/mTORC2 & $\begin{array}{c}(-) /(+) \\
(\mathrm{n}=17)\end{array}$ & $\begin{array}{c}(-) /(-),(+) /(-),(+) /(+) \\
(\mathrm{n}=55)\end{array}$ & $p$-Value \\
\hline VEGF-A & & & \\
Positive & 7 & 43 & $p<0.05$ \\
Negative & 10 & 12 & \\
VEGF-C & & & \\
Positive & 9 & 42 & \\
Negative & 8 & 13 & \\
HIF- $\alpha$ & & & \\
Positive & 7 & 43 & $p<0.05$ \\
Negative & 10 & 12 & \\
\hline
\end{tabular}

$(-) /(+)$ : mTORC1(-)mTORC2(+) group; (-)/(-): mTORC1(-)mTORC2(-) group; $(+) /(-)$ : mTORC1 $(+)$ mTORC2 $(-)$ group; $(+) /(+): \operatorname{mTORC} 1(+)$ mTORC2(+) group. 
regulation and functions of $\mathrm{mTORC} 2$ are less well understood. mTORC2 was first reported to be involved in the control of actin cytoskeleton organization (31). Subsequent studies have suggested that mTORC2 also plays a part in the regulation of proliferation, survival, and nutrient uptake in cancer cells (3234). In the present study, both mTORC1 and mTORC2 expression levels correlated with T stage and PCNA expression. Hence, we suggested that mTORC1 and mTORC2 play roles in the cell proliferation of OSCC. In addition, in present study, we demonstrated that cases of mTORC1(-)/mTORC2(+) had significantly lower expression levels of VEGFs and HIF1- $\alpha$ compared with the other expression pattern groups.

VEGF-A overexpression has been reported in most types of cancer, including oral cancer, and it is thought to be a prognostic factor for survival (2). VEGF-C has been detected in several different types of cancer, and its level in some studies seemed to correlate with nodal metastasis and patient survival (2). The HIF- $1 \alpha$ transcription factor plays an essential role in oxygen homeostasis, and high expression of HIF-1 $\alpha$ protein has been found to be associated with both tumor aggressiveness and an unfavorable prognosis in various types of cancer (2). Hence, we suggest mTORC1(-)/mTORC2(+) may have a low potential for malignancy in OSCC, and furthermore have correlation with the effectiveness of anti-mTOR drugs.

\section{Conclusion}

mTORC1 and mTORC2 were found to be overexpressed in OSCC, and they were significantly correlated with clinicopathological factors. In a previous study, we concluded that expression levels of mTORC1-HIF1-VEGF had a significant correlation with the effects of anti-mTOR drugs. In the present study, the mTORC1 $(-) /$ mTORC2 (+) group showed significantly lower VEGF and HIF1 expression levels. Hence, it was suggested that mTORC1 and mTORC 2 could be promising targets for anti-tumor effects in OSCC, and mTORC1 (-)/mTORC2 (+) may have a correlation with the effectiveness of anti-mTOR drugs.

\section{References}

1 Shaw RJ and Cantley LC: Ras, PI(3)K and mTOR signaling controls tumor cell growth. Nature 441: 424-430, 2006.

2 Naruse T, Kawasaki G, Yanamoto S, Mizuno A and Umeda M: Immunohistochemical study of VEGF expression in oral squamous cell carcinomas: correlation with the mTOR-HIF-1 pathway. Anticancer Res 31: 4429-4438, 2011.

3 Zaytseva YY, Valentino JD, Gulhati P and Evers BM: mTOR inibtors in cancer therapy. Cancer Lett 319: 1-7, 2012.

4 Liu FY, Zhao ZJ, Li P, Ding X, Zong ZH and Sun CF: Mammalian target of rapamycin (mTOR) is involved in the survival of cells mediated by chemokine receptor 7 through $\mathrm{PI} 3 \mathrm{~K} / \mathrm{Akt}$ in metastatic squamous cell carcinoma of the head and neck. Br J Oral Maxillofac Surg 48: 291-296, 2010.
5 Laplante $\mathrm{M}$ and Sabatini DM: mTOR signaling in growth control and disease. Cell 149: 274-293, 2012.

6 Strimpakos AS, Karapanagitou EM, Saif MF and Syrigos KN: The role of mTOR in the management of solid tumors: an overview. Cancer Treat Rev 35: 148-159, 2009.

7 Huo HZ, Zhou ZY, Wang B, Qin J, Liu WY and Gu Y: Dramatic suppression of colorectal cancer cell growth by the dual mTORC1 and mTORC2 inhibitor AZD-2014. Biochem Biophys Res Commun 443: 406-412, 2014.

8 Laplante $\mathrm{M}$ and Sabatini DM: mTOR signaling at a glance. J Cell Sci 122: 3589-3594, 2009.

9 Li C, Cui JF, Chen MB, Liu CY, Liu F, Zhang QD, Zou J and Lu PH: The preclinical evaluation of the dual mTORC1/2 inhibitor INK-128 as a potential anti-colorectal cancer agent. Cancer Biol Ther 16: 34-42, 2015.

10 Van Duyne GD, Standaert RF, Karplus PA, Schreiber SL and Clardy J: Atomic structures of the human immunophilin FKBP12 complexes with FK506 and rapamycin. J Med Biol 229: 105124, 1993.

11 Marques AEM, Elias ST, Porporatti AL, Castilho RM, Squarize $\mathrm{CH}$, Canto GDL and Guerra ENS: mTOR pathway protein immunoexpression as a prognostic factor for survival in head and neck cancer patients: a systematic review and meta-analysis. J Oral Pathol Med 45: 319-328, 2016.

12 Monteiro LS, Delgado ML, Ricardo S, Garcez F, Amaral BD, Warnakulasuriya $\mathrm{S}$ and Lopes C: Phosphorylated mammalian target of rapamycin is associated with adverse outcome in oral squamous cell carcinoma. Oral Surg Oral Med Oral Pathol Oral Radiol 115: 638-645, 2013

13 Zou L, Huang Y, Li J and Wang Z: The mTOR pathway is associated with the poor prognosis of human hepatocellular carcinoma. Med Oncol 27: 255-261, 2010.

14 Sun $\mathrm{CH}$, Chang $\mathrm{YH}$ and Pan $\mathrm{CC}$ : Activation of the $\mathrm{PI} 3 \mathrm{~K} / \mathrm{Akt} / \mathrm{mTOR}$ pathway correlates with tumor progression and reduced survival in patients with urothelial carcinoma of the urinary bladder. Histopathology 58: 1054-1063, 2011.

15 Sobin $\mathrm{LH}$ and Wittekind $\mathrm{CH}$ : International Union Against Cancer: TNM Classification of Malignant Tumours, 5th edn., New York, Wiley, pp. 1-277, 1997.

16 Wahi PN: (WHO) Histological Typing of Oral and Oropharyngeal Tumours, 4th edn. Geneva, WHO, pp. 1-28, 1971.

17 Yamamoto K, Kohama G, Sunakawa H, Iwai M and Hiratsuka $\mathrm{H}$ : Mode of invasion, bleomycin sensitivity, and clinical course in squamous cell carcinoma of the oral cavity. Cancer 51: 21752180, 1983.

18 Houchens DP, Ovejera AA, Riblet SM and Slagel DE: Human brain tumor xenografts in nude mice as a chemotherapy model. Eur J Cancer Clin Oncol 19: 799-805, 1983.

19 Naruse T, Yanamoto S, Yamada S, Rokutanda S, Kawakita A, Kawasaki G and Umeda M: Anti-tumor effect of the mammalian target of rapamycin inhibitor everolimus in oral squamous cell carcinoma. Pathol Oncol Res 21: 765-773, 2015.

20 Hardt M, Chantaravisoot $\mathrm{N}$ and Tamanoi F: Activating mutations of TOR (target of ramamycin). Genes Cells 16: 141-151, 2011.

21 Gulhati P, Cai Q, Li J, Liu J Rychahou PG, Qui S, Lee EY, Silva SR, Bowen KA, Gao $\mathrm{T}$ and Evers BM: Targeted inhibition of mammalian target of rapamycin signaling inhibits tumorigenesis of colorectal cancer. Clin Cancer Res 15: 72077216, 2009. 
22 Chiang GG and Abraham RT: Targeting the mTOR signaling network in cancer. Trends Mol Med 13: 433-442, 2007.

23 Roulin D, erantola Y, Dormond-Meuwhly A, Demartines N, and Dormond O: Targeting mTORC2 inhibits colon cancer cell proliferation in vitro and tumor formation in vivo. Mol Cancer 9: $57,2010$.

$24 \mathrm{Wu}$ WK, Lee CW, Cho CH, Chan FK, Yu J and Sung JJ: RNA interference targeting raptor inhibits proliferation of gastric cancer cells. Exp Cell Res 317: 1353-1358, 2011.

25 Masri J, Bernath A, Martin J, Jo OD, Vartanian R, Funk A and Gera J: mTORC2 activity is elevated in gliomas and promotes growth and cell motility via overexpression of rictor. Cancer Res 67: 11712-11720, 2007.

26 Gulhati P, Bowen KA, Liu J, Stevens PD, Rychahou PG, Chen M, Lee EY, Weiss HL, O'Coner KL, Gao T and Evers BM: mTORC1 and mTORC2 regulate EMT, motility, and metastasis of colorectal cancer via RhoA and Rac1 signaling pathways. Cancer Res 71: 3246-3256, 2011.

27 Zhang F, Zhang X, Li M, Chen P, Zhang B, Guo H, Cao W, Wei $\mathrm{X}$, Cao X, Hao X and Zhan N: mTOR complex component Rictor interacts with PKCzeta and regulates cancer cell metastasis. Cancer Res 70: 90360-9370, 2010.

28 Patel V, Marsh CA, Dorsam RT, Mikelis CM, Masedunskas A, Amornphimoltham P, Nathan CA, Singh B, Weight R, Molinolo AA and Gutkind JS: Decreased lymphangiogenesis and lymph node metastasis by mTOR inhibition in head and neck cancer. Cancer Res 71: 7103-7102, 2011.
29 Kim DH and Sabatini DM: Raptor and mTOR: subunits of a nutrient-sensitive complex. Cur Top Microbiol Immunol 279: 259-270, 2004.

30 Yecies JL and Manning BD: Transcriptional control of cellular metabolism by mTOR signaling. Cancer Res 71: 2815-2820, 2011

31 Foster KG and Finger DC: Mammalian target of rapamycin (mTOR): conducting the cellular signaling symphony. J Biol Chem 285: 14071-14077, 2010.

32 Zoncu R, Efeyan A and Sabatini DM: MTOR: from growth signal integration to cancer, diabetes and aging. Nat Rev Mol Cell Biol 12: 21-35, 2011.

33 Maeto J, Oimos D, Dumez H, PoondruS, Samberg NL, Barr S, Van-Tomout JM, Jie F, Sandhu S, Tan DS, Moreno V, LoRusso PM, Kaye SB and Schoffski P: A first I n man, dose-finding study of the mTORC1/mTORC2 inhibitor OSI-027 in patients with advanced solid malignancies. Br J Cancer 114: 889-896, 2016.

34 Bibi Z, Ahmed J, Siddiqa A, Paracha RZ, Saeed T, Ali A, Janjua HA, Ullah S, Abdallah EB and Roux O: Formal modeling of mTOR associated biological regulatory network reveals novel therapeutic strategy for the treatment of cancer. Front Physiol 8: 416-429, 2017.
Received November 13, 2017

Revised December 18, 2017

Accepted December 20, 2017 